\title{
Dissociative production of vector mesons within the energy-dependent hot-spot model
}

\section{Bendova*, J. Cepila, and J. G. Contreras}

Faculty of Nuclear Sciences and Physical Engineering, Czech Technical University in Prague, Prague 11519, Czech Republic

E-mail: dagmar.bendova@fjfi.cvut.cz, jan.cepila@fjfi.cvut.cz, jgcn@mail.cern.ch

\begin{abstract}
We present a model with which we predict the cross sections for the exclusive and the dissociative photo- and electroproduction of light and heavy vector mesons off protons; the model describes correctly available experimental data. The model is based on the color-dipole approach and incorporates geometric fluctuations of the target-proton partonic structure. The fluctuations are generated as randomly placed areas of high gluonic density, so-called hot spots, in the impactparameter plane, with the number of hot spots being energy-dependent. A striking feature of the model is the prediction of a maximum of the dissociative cross section as a function of the centerof-mass energy $W_{\gamma p}$, followed by a steep decrease as the hot spots start to overlap with increasing energy.
\end{abstract}

European Physical Society Conference on High Energy Physics - EPS-HEP2019 -

10-17 July, 2019

Ghent, Belgium

${ }^{*}$ Speaker. 


\section{Introduction}

According to the predictions of quantum chromodynamics (QCD), the partonic structure of hadrons evolves linearly with energy or, equivalently, with Bjorken- $x$. However at some point, it is predicted that this behavior changes and nonlinear effects start to manifest themselves and the hadron enters a so called saturated regime; for a review see e.g. [1]. The production of vector mesons has been considered to serve as a good probe of proton saturation at LHC [2] and at future EIC facilities [3, 4]. In the exclusive process, the incoming electron emits a photon which interacts with the proton with the subsequent production of a vector meson, while the proton remains intact. The photon can be either quasi-real or it can have a large virtuality $Q^{2}$, we refer to these cases as photo- and electroproduction, respectively. In the related dissociative process, a target proton is broken into a hadronic system. In a Good-Walker approach [5, 6], this process can be related to fluctuations of the partonic structure of the proton. This approach has been used to advocate that large-scale fluctuations of the partonic structure are necessary to obtain a correct description of the $\mathrm{J} / \psi$ photoproduction cross section as a function of $|t|$, which is the square of the momentum transfer of the proton, at a fixed center-of-mass energy $W_{\gamma p}$ of the photon-proton system. The GoodWalker approach has also been applied in [2] to predict a maximum of the total dissociative $\mathrm{J} / \psi$ photoproduction cross section as a function of $W_{\gamma \mathrm{p}}$, using a so called energy-dependent hot-spot model. These studies were continued in [7] to study the mass dependence of the dissociative cross section for various vector mesons and in [8] to study the dissociative electroproduction of vector mesons and the scale dependence of the predicted geometrical saturation. This proceedings review the predictions of [8] for the exclusive and the dissociative production of vector mesons. The text is organized as follows. In Sec. 2, we give a brief description of the formalism, in Sec. 3, we present the predictions of our model and compare them with the available experimental data, and in Sec. 4 , we give a brief conclusion of the results.

\section{Formalism of the vector meson production}

The exclusive cross section to produce a vector meson $\mathrm{V}$ is given by

$$
\left.\frac{\mathrm{d} \sigma^{\gamma^{*} p \rightarrow \mathrm{V} p}}{\mathrm{~d}|t|}\right|_{T, L}=\frac{\left(R_{g}^{T, L}\right)^{2}}{16 \pi}\left|\left\langle\mathscr{A}_{T, L}\right\rangle\right|^{2},
$$

where the average is over different target configurations. The dissociative cross section is given as a variance over different configurations

$$
\left.\frac{\mathrm{d} \sigma^{\gamma^{*} p \rightarrow \mathrm{V} Y}}{\mathrm{~d}|t|}\right|_{T, L}=\frac{\left(R_{g}^{T, L}\right)^{2}}{16 \pi}\left(\left\langle\left|\mathscr{A}_{T, L}\right|^{2}\right\rangle-\left|\left\langle\mathscr{A}_{T, L}\right\rangle\right|^{2}\right),
$$

where $R_{g}$ is called the skewedness correction [9] and $\mathscr{A}_{T, L}$ is the photon-proton scattering amplitude. The production of a vector meson can be described within the color dipole picture [10,11], in which the incoming photon fluctuates into a $q \bar{q}$ dipole which interacts with the target proton in 
its rest frame. In this formalism, the scattering amplitude can be expressed as

$$
\mathscr{A}_{T, L}\left(x, Q^{2}, \vec{\Delta}\right)=i \int \mathrm{d} \vec{r} \int_{0}^{1} \frac{\mathrm{d} z}{4 \pi} \int \mathrm{d} \vec{b}\left|\Psi_{\mathrm{V}}^{*} \Psi_{\gamma^{*}}\right|_{T, L} \exp [-i(\vec{b}-(1-z) \vec{r}) \vec{\Delta}] \frac{\mathrm{d} \sigma_{q \bar{q}}}{\mathrm{~d} \vec{b}}
$$

where $T, L$ denote transverse and longitudinal case, respectively, $x$ denotes the Bjorken- $x$ of the exchanged pomeron, $\vec{\Delta}^{2} \equiv-t, \vec{r}$ is the transverse size of the color dipole, $z$ is the fraction of the photon longitudinal momentum carried by the quark, $\vec{b}$ is the impact parameter between the dipole and the proton, and $\Psi_{\mathrm{V}}, \Psi_{\gamma^{*}}$ are the wave functions of the vector meson and a virtual photon, respectively. The dipole-proton cross section $\frac{\mathrm{d} \sigma_{q \bar{q}}}{\mathrm{~d} \vec{b}}$ can be expressed, in the impact-parameter factorized form, as

$$
\frac{\mathrm{d} \sigma_{q \bar{q}}}{\mathrm{~d} \vec{b}}=\sigma_{0} N(x, r) T_{p}(\vec{b})
$$

where the function $T_{p}(\vec{b})$ describes the proton profile in the impact-parameter plane, $\sigma_{0}$ is a normalization parameter, and $N(x, \vec{r})$ is the dipole scattering amplitude.

In the energy-dependent hot-spot model [2], the proton profile is seen as a set of regions of high gluon density (hot spots). The positions of these hot spots in the impact-parameter plane fluctuate event-by-event and are described by the proton profile function

$$
T_{p}(\vec{b})=\frac{1}{N_{h s}} \sum_{i=1}^{N_{h s}} T_{h s}\left(\vec{b}-\vec{b}_{i}\right)
$$

where each hot spot is defined by

$$
T_{h s}\left(\vec{b}-\vec{b}_{i}\right)=\frac{1}{2 \pi B_{h s}} \exp \left(-\frac{\left(\vec{b}-\vec{b}_{i}\right)^{2}}{2 B_{h s}}\right)
$$

where $B_{p}$ and $B_{h s}$ are related to the radius of the proton and hot spot, respectively. The energyevolution of the transverse structure of the proton is ensured by the number of hot spots $N_{h s}$ increasing with decreasing $x$ as

$$
\left\langle N_{h s}(x)\right\rangle=p_{0} x^{p_{1}}\left(1+p_{2} \sqrt{x}\right),
$$

where $p_{0}, p_{1}$, and $p_{2}$ are model parameters. The description, specific values, and the detailed discussion of the model parameters, including also a description of the approach to the vector meson and photon wave functions and the parametrization of the scattering amplitude $N(x, \vec{r})$ are described in $[2,7,8]$.

\section{Predictions and comparison with data}

Using the model briefly described above, we predict an energy $W_{\gamma \mathrm{p}}$ dependence of the exclusive and dissociative photo- and electroproduction of vector mesons off the proton target. In this contribution, we show the predictions for $\rho, \phi, \mathrm{J} / \psi$, and $\Upsilon(1 S)$ mesons, the predictions for $\psi(2 S)$ and $\Upsilon(2 S)$ are shown in [8]. The predictions are compared with the available experimental data. 
The predictions for the energy $W_{\gamma p}$ dependence of the exclusive and dissociative total cross sections for a $\rho$ meson are shown in Fig. 1. We compare these predictions with CMS data [12] for $\rho$ photoproduction in $\mathrm{p}-\mathrm{Pb}$ collisions. We also compare the predictions with HERA data $[13,14,15$, $16,17]$ for several values of $Q^{2}$. The predictions for exclusive photoproduction and both exclusive and dissociative electroproduction at $Q^{2}$ from 2.4 to $35.6 \mathrm{GeV}^{2}$ give a successful description of the available experimental data. The $\mathrm{H} 1$ collaboration has recently presented preliminary data (not shown in Fig. 1) for dissociative photoproduction of $\rho$ meson. Our predictions also agree with these data, however final conclusions can be made after the data are officially published.
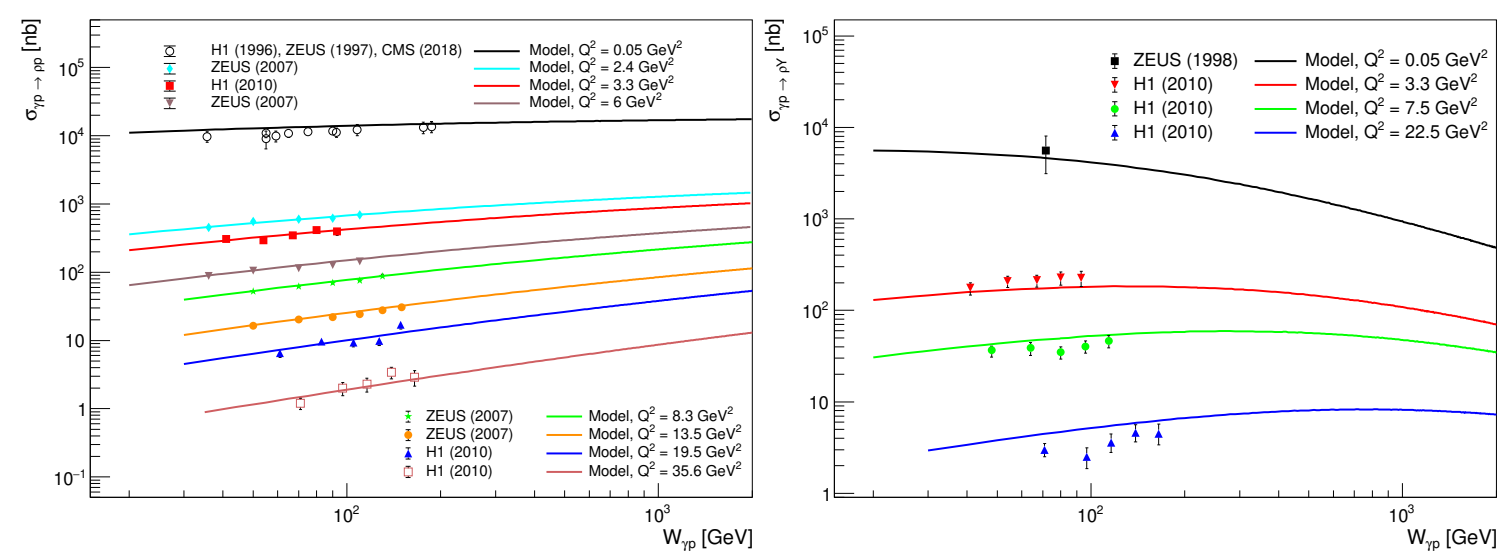

Figure 1: Comparison of the model predictions (solid lines) with HERA [13, 14, 15, 16, 17] and CMS data [12] for the $W_{\gamma p}$ dependence of the exclusive (left) and dissociative (right) photo- and electroproduction cross section of a $\rho$ meson. Figures taken from [8].
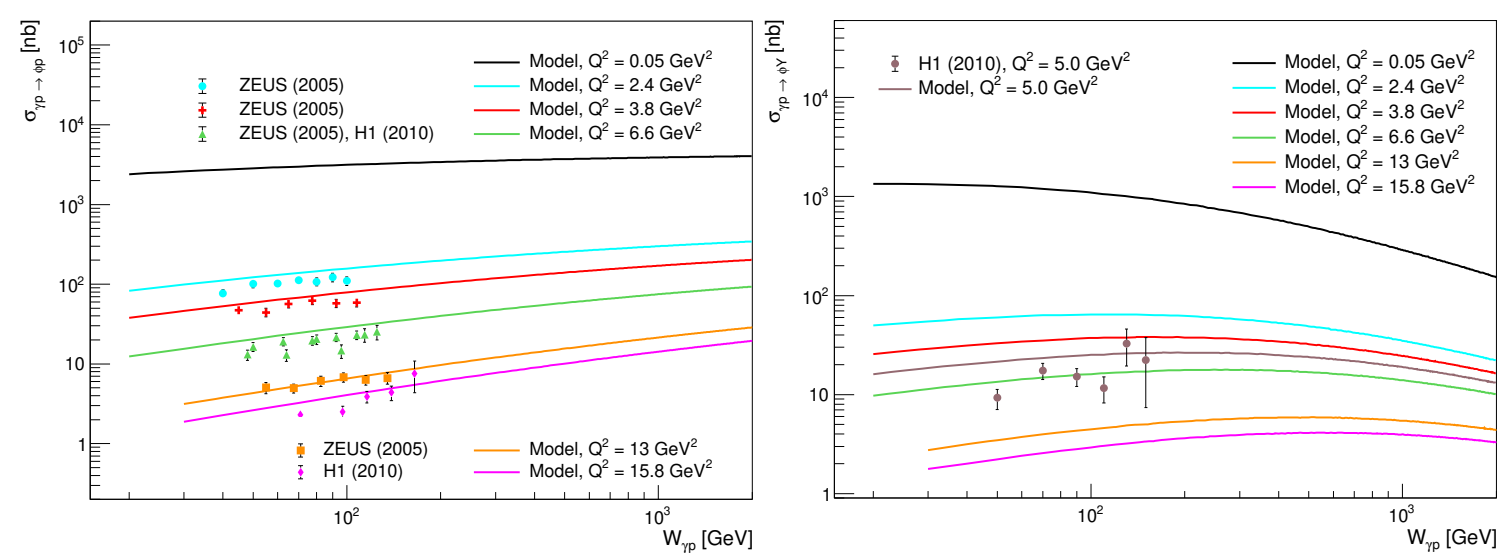

Figure 2: Comparison of the model predictions (solid lines) with HERA data from H1 [14, 17] and ZEUS [18] for the $W_{\gamma p}$ dependence of the exclusive (left) and dissociative (right) photo- and electroproduction cross section of a $\phi$ meson. Figures taken from [8].

The predictions for the $W_{\gamma p}$ dependence of the exclusive and dissociative cross sections for a $\phi$ meson are shown in Fig. 2 and compared with the data from H1 [14, 17] and ZEUS [18]. The agreement between the model and the data is satisfactory, especially for exclusive electroproduction, however it is not as good as in the case of $\rho$ meson. 

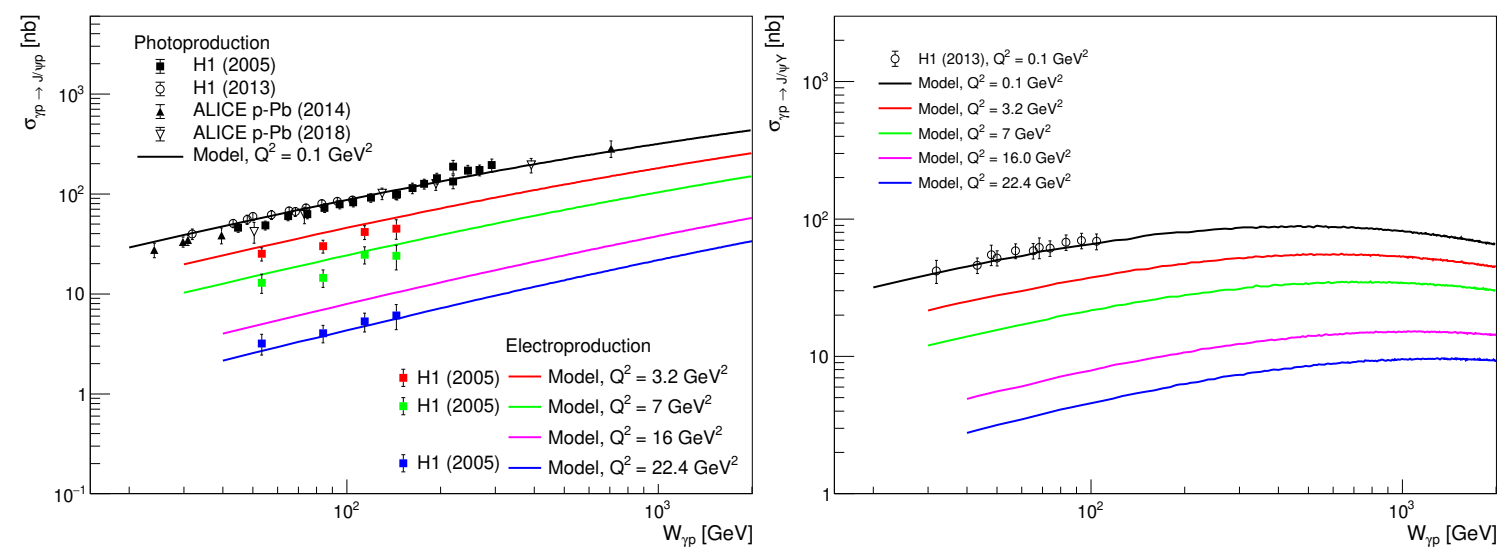

Figure 3: Comparison of the model predictions (solid lines) with HERA data from H1 [19, 20] and LHC data from ALICE [21, 22] for the $W_{\gamma p}$ dependence of the exclusive (left) and dissociative (right) photo- and electroproduction cross section of a $J / \psi$ meson. Figures taken from [8].

The predictions of the model for the production of a $J / \psi$ meson are shown in Fig. 3. The photoproduction process, both exclusive and dissociative, has already been studied in previous works [2, 7]. For completeness, the same comparison with H1 [19, 20] and ALICE data [21] from ultraperipheral $\mathrm{p}-\mathrm{Pb}$ collisions at $\sqrt{s}=5.02 \mathrm{TeV}$ is shown together with recent ALICE data [22], all the available data are successfully described by the model predictions. Predictions for exclusive electroproduction also correctly describe available data from H1 [19]. Unfortunately, there is no data for the dissociative process to compare with.

The predictions for exclusive and dissociative cross section for an $\Upsilon(1 S)$ meson have also been studied in [7]. Here we show them again in Fig. 4 together with the electroproduction predictions to provide the comparison of both processes. We compare the exclusive $\Upsilon(1 S)$ photoproduction cross section prediction with data from HERA measured at H1 [23] and ZEUS [24] and also with data from the LHC measured at the LHCb [25] and with new data from CMS [26]. The agreement of the model predictions with the data is good, however the large uncertainties of the data prevent us from making any final conclusion.

For completeness, we have also calculated predictions for the exclusive and dissociative production of $\psi(2 S)$ and $\Upsilon(2 S)$ mesons. They are not shown in this contribution, both the predictions and the comparison with the data for $\psi(2 \mathrm{~S})$ to $\mathrm{J} / \psi$ ratios are discussed in [8].

\section{Conclusions}

In this contribution, we have presented the predictions for exclusive and dissociative photoand electroproduction of vector mesons $\rho, \phi, \mathrm{J} / \psi$, and $\Upsilon(1 S)$ off protons using the energy-dependent hot-spot model. We observe that the total exclusive cross section grows with increasing energy $W_{\gamma p}$. However, the dissociative process shows a striking behavior. We observe that the cross section rises at low energies and at some point, it reaches a maximum, after which it is decreasing as a function of increasing $W_{\gamma p}$. This behavior is caused by the variance over the different configurations, when the available area in the impact-parameter plane of the proton is filled with hot spots, they start to overlap and all the possible configurations look alike which causes the variance to decrease. 

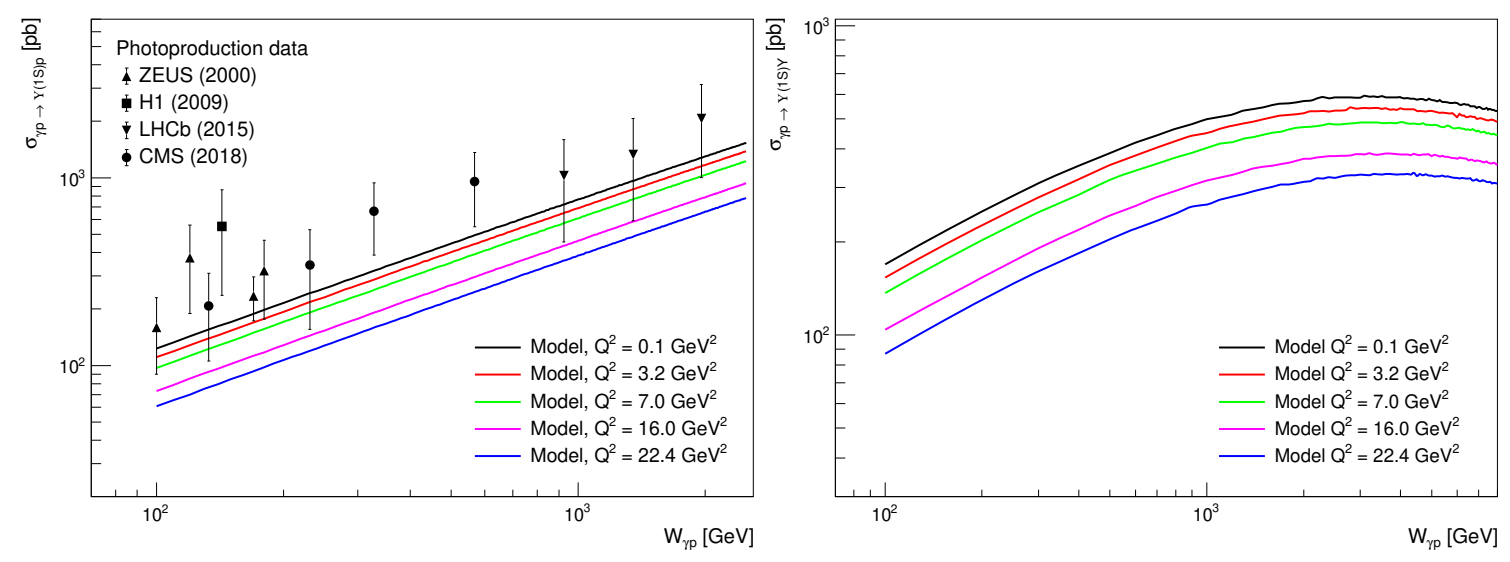

Figure 4: Comparison of the model predictions (solid lines) with HERA data from H1 [23] and ZEUS [24], and with the LHC data from LHCb [25] and CMS [26] for the $W_{\gamma p}$ dependence of the exclusive (left) and dissociative (right) photo- and electroproduction cross section of an $\Upsilon(1 S)$ meson. Figures taken from [8].

This behavior of the total dissociative cross section is clearly present for all vector mesons, with the position of the maximum in $W_{\gamma p}$ being shifted to higher energies with increasing mass of the vector meson and the $Q^{2}$ of the exchanged photon. This phenomenon arises from the geometrical fluctuations of the proton in the transverse plane and could be studied at future EIC facilities.

\section{Acknowledgements}

This work has been supported by the COST Action CA15213 THOR, by the grant LTC17038 of the INTER-EXCELLENCE program at the Ministry of Education, Youth and Sports of the Czech Republic, and Grant No. 17-04505S of the Czech Science Foundation (GACR).

\section{References}

[1] F. Gelis, E. Iancu, J. Jalilian-Marian and R. Venugopalan, The Color Glass Condensate, Ann. Rev. Nucl. Part. Sci. 60 (2010) 463 [1002.0333].

[2] J. Cepila, J. G. Contreras and J. D. Tapia Takaki, Energy dependence of dissociative J/ $\psi$ photoproduction as a signature of gluon saturation at the LHC, Phys. Lett. B766 (2017) 186 [1608.07559].

[3] A. Accardi et al., Electron Ion Collider: The Next QCD Frontier, Eur. Phys. J. A52 (2016) 268 [1212.1701].

[4] LHeC Study Group collaboration, A Large Hadron Electron Collider at CERN: Report on the Physics and Design Concepts for Machine and Detector, J. Phys. G39 (2012) 075001 [1206.2913].

[5] M. L. Good and W. D. Walker, Diffraction disssociation of beam particles, Phys. Rev. 120 (1960) 1857.

[6] H. I. Miettinen and J. Pumplin, Diffraction Scattering and the Parton Structure of Hadrons, Phys. Rev. D18 (1978) 1696.

[7] J. Cepila, J. G. Contreras, M. Krelina and J. D. Tapia Takaki, Mass dependence of vector meson photoproduction off protons and nuclei within the energy-dependent hot-spot model, Nucl. Phys. B934 (2018) 330 [180 . 05508]. 
[8] D. Bendova, J. Cepila and J. G. Contreras, Dissociative production of vector mesons at electron-ion colliders, Phys. Rev. D99 (2019) 034025 [1811.06479].

[9] A. G. Shuvaev, K. J. Golec-Biernat, A. D. Martin and M. G. Ryskin, Off diagonal distributions fixed by diagonal partons at small $x$ and xi, Phys. Rev. D60 (1999) 014015 [hep-ph/9902410].

[10] A. H. Mueller, Soft gluons in the infinite momentum wave function and the BFKL pomeron, Nucl. Phys. B415 (1994) 373.

[11] A. H. Mueller and B. Patel, Single and double BFKL pomeron exchange and a dipole picture of high-energy hard processes, Nucl. Phys. B425 (1994) 471 [hep-ph/ 9403256 ].

[12] CMS collaboration, Measurement of exclusive $\rho(770)^{0}$ photoproduction in ultraperipheral $p P b$ collisions at $\sqrt{s_{\mathrm{NN}}}=5.02 \mathrm{TeV}$, Eur. Phys. J. C79 (2019) 702 [1902.01339].

[13] H1 collaboration, Elastic photoproduction of rho0 mesons at HERA, Nucl. Phys. B463 (1996) 3 [hep-ex/9601004].

[14] H1 collaboration, Proton dissociative $\rho$ and elastic $\phi$ electroproduction at HERA, Z. Phys. $\mathbf{C 7 5}$ (1997) 607 [hep-ex/9705014].

[15] ZEUS collaboration, Elastic and proton dissociative $\rho^{0}$ photoproduction at HERA, Eur. Phys. J. C2 (1998) 247 [hep-ex/9712020].

[16] ZEUS collaboration, Exclusive rho0 production in deep inelastic scattering at HERA, PMC Phys. A1 (2007) 6 [0708.1478].

[17] H1 collaboration, Diffractive Electroproduction of rho and phi Mesons at HERA, JHEP 05 (2010) $032[0910.5831]$.

[18] ZEUS collaboration, Exclusive electroproduction of phi mesons at HERA, Nucl. Phys. B718 (2005) 3 [hep-ex/0504010].

[19] H1 collaboration, Elastic J/psi production at HERA, Eur. Phys. J. C46 (2006) 585 [hep-ex/0510016].

[20] H1 collaboration, Elastic and Proton-Dissociative Photoproduction of J/psi Mesons at HERA, Eur. Phys. J. C73 (2013) 2466 [1304.5162].

[21] ALICE collaboration, Exclusive J/ $\psi$ photoproduction off protons in ultra-peripheral p-Pb collisions at $\sqrt{s_{\mathrm{NN}}}=5.02$ TeV, Phys. Rev. Lett. 113 (2014) 232504 [1 406.7819 ].

[22] ALICE collaboration, Energy dependence of exclusive $\mathrm{J} / \psi$ photoproduction off protons in ultra-peripheral p-Pb collisions at $\sqrt{s_{\mathrm{NN}}}=5.02$ TeV, Eur. Phys. J. C79 (2019) 402 [1809. 03235 ].

[23] H1 collaboration, Elastic photoproduction of J / psi and Upsilon mesons at HERA, Phys. Lett. B483 (2000) 23 [hep-ex/0003020].

[24] ZEUS collaboration, Exclusive photoproduction of upsilon mesons at HERA, Phys. Lett. $\mathbf{B 6 8 0}$ (2009) 4 [0903.4205].

[25] LHCB collaboration, Measurement of the exclusive $\Upsilon$ production cross-section in pp collisions at $\sqrt{s}=7 \mathrm{TeV}$ and $8 \mathrm{TeV}, \mathrm{JHEP} 09$ (2015) 084 [1505.08139].

[26] CMS collaboration, Measurement of exclusive $\Upsilon$ photoproduction from protons in pPb collisions at $\sqrt{s_{\mathrm{NN}}}=5.02 \mathrm{TeV}$, Eur. Phys. J. C79 (2019) 277 [1809.11080]. 